\title{
Simultaneous choice of time points and the block design in the growth curve model
}

\author{
Anna Szczepańska
}

Received: 31 March 2011 / Revised: 15 February 2012 / Published online: 20 March 2012

(C) The Author(s) 2012. This article is published with open access at Springerlink.com

\begin{abstract}
The aim of this paper is to consider the optimality in the growth curve model with respect to two aspects: time and the block design and to show some relations between information functions for different designs. The A-, D- and E-optimality are studied.
\end{abstract}

Keywords Growth curve model - Information matrix · Information function · Optimal design · A-, D- and E-optimality

\section{Introduction}

An experiment where observations are taken in time can be described by the growth curve model. This model was proposed by Potthoff and Roy (1964) and later their theory was expanded on by many authors. The aim of this paper is to analyse the experiment based on the growth curve model which is conducted in the block design and the optimality of design is studied. In the literature optimal designs in multivariate models was considered by Markiewicz and Szczepańska (2007), and Filipiak et al. (2009). The authors determined optimal designs with respect to an allocation of treatments in blocks in the case where the dispersion matrix of measurements was known or unknown. They showed the relation between optimal designs in univariate models and their multivariate extensions. The optimal choice of time points of measurements in the experiment was considered by Moerbeek (2005), Filipiak and Szczepańska (2005, 2007). Using A-, D-, E-criteria of optimality and assuming different

\footnotetext{
A. Szczepańska $(\varangle)$

Department of Mathematical and Statistical Methods, Poznań University of Life Sciences, Wojska Polskiego 28, 60637 Poznań, Poland e-mail: sanna6@wp.pl
} 
correlations between observations, authors determined the optimal allocation of time points in the given interval.

The aim of this paper is to consider the optimal choice of time points and the block design in the experiment and show some relations between information functions for different designs.

We have organized the paper as follows. In Sect. 2 the model of experiment is considered and the form of the information matrix for the estimation of the treatment effects in this model is given. In Sect. 3 optimality criteria for designs are formulated. Some results about the optimality with respect to an allocation of time points and an allocation of treatments in the experiment are shown in Sect. 4. In Sect. 5 some relations for information functions for different designs are given. The last section contains the discussion part where some limitations of the present study are presented and the possibility of future research is given.

\section{Extended growth curve model}

Consider an experiment in the block design where treatments are arranged in $n=b k$ plots where $b$ denotes the number of blocks and $k$ is the size of each block. The illustration of this experiment is as follows

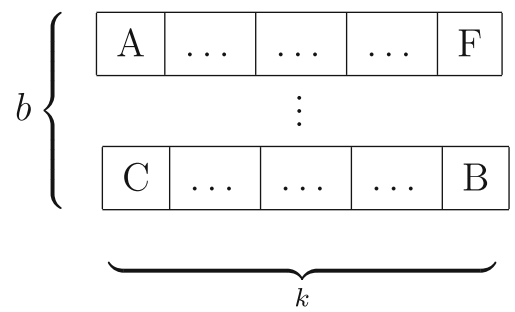

The material within the blocks is relatively homogeneous but it differs between blocks. Suppose that in the block design the characteristic is measured at $q$ time points which are denoted by $l_{j}, j=1,2, \ldots, q$ and observations for $v$ treatments are compared in time. Assume that observations on each plot are taken once at each time point, and observations on all plots are taken at the same time points. In this experiment we consider two kinds of designs: design $t \in \mathcal{T}$ and design $d \in \mathcal{D}$ which mean an allocation of time points in the experiment and an allocation of treatments in the block design, respectively. Thus, $\mathcal{T}$ denotes the class of sets of time points chosen from the given time interval: $[a, b], t=\left\{l_{1}, l_{2}, \ldots, l_{q}\right\}, t \in \mathcal{T}$, where $l_{j} \in[a, b], j=1,2, \ldots, q$. Class $\mathcal{D}$ denotes the class of block designs with $v$ treatments, $b$ blocks of size $k$. The different allocation of treatments in blocks means the different block design in $\mathcal{D}$. The block design is described by the matrix where rows denote blocks and the number of columns is equal to the size of blocks. Elements of the matrix are labels of treatments.

The extended growth curve model in the experiment on the block design has the form of

$$
\mathbf{Y}=\mathbf{A}_{1, d} \mathbf{B}_{1} \mathbf{C}_{1, t}+\mathbf{A}_{2} \mathbf{B}_{2} \mathbf{C}_{1, t}+\mathbf{E}, \quad \mathbf{E} \sim N_{n, q}\left(\mathbf{0}, \mathbf{\Sigma} \otimes \mathbf{I}_{n}\right),
$$


where $\mathbf{Y}$ is the $n \times q$ matrix of observations, the $n \times v$ matrix $\mathbf{A}_{1, d}$ is the design matrix of treatment effects, the $n \times b$ matrix $\mathbf{A}_{2}=\mathbf{I}_{b} \otimes \mathbf{1}_{k}$ is the design matrix of block effects, where $\mathbf{I}_{b}$ is the $b \times b$ identity matrix and $\mathbf{1}_{k}$ means the vector of 1 's. The matrix $\mathbf{A}_{1, d}$ is indexed by $d$ because it changes for the different allocation of treatments in blocks. The $v \times(p+1)$ matrix $\mathbf{B}_{1}$, the $b \times(p+1)$ matrix $\mathbf{B}_{2}$ are matrices of unknown parameters of treatment effects and block effects, respectively. The $(p+1) \times q$ matrix $\mathbf{C}_{1, t}$ is the design matrix of time points, where $p$ denotes a degree of polynomial, and $\mathbf{E}$ is the $n \times q$ matrix of random errors with the mean equal to zero. We assume that in the block observations of plots on different treatments measured at the same time points are uncorrelated, while the correlation between observations of plots at different time points is described by the matrix $\boldsymbol{\Sigma}$. Hence, the dispersion matrix of errors has the form $D(\mathbf{E})=\boldsymbol{\Sigma} \otimes \mathbf{I}_{n}$, where $\boldsymbol{\Sigma}$ is the known $q \times q$ positive definite matrix and the symbol $\otimes$ denotes the Kronecker product.

In the considered model the design matrix of time points, $\mathbf{C}_{1, t}$, is the type of Vandermond matrix of the form

$$
\mathbf{C}_{1, t}=\left[\begin{array}{ccccc}
1 & 1 & 1 & \cdots & 1 \\
l_{1} & l_{2} & l_{3} & \cdots & l_{q} \\
\vdots & \vdots & \vdots & \ddots & \vdots \\
l_{1} p & l_{2} p & l_{3} p & \cdots & l_{q}^{p}
\end{array}\right] .
$$

Model (1) can be also written as

$$
\mathbf{Y}=\left(\mathbf{A}_{1, d} \mathbf{B}_{1}+\mathbf{A}_{2} \mathbf{B}_{2}\right) \mathbf{C}_{1, t}+\mathbf{E}, \quad \mathbf{E} \sim N_{n, q}\left(\mathbf{0}, \boldsymbol{\Sigma} \otimes \mathbf{I}_{n}\right)
$$

and it is the extension of the model given by Potthoff and Roy (1964). However in this model one matrix of curve coefficients can be estimated and its elements are sums of treatment effects and block effects. In our considerations only the estimation of treatment effects is interesting so the form of model (1) is more appropriate.

Assuming that the matrix of random errors has the normal distribution and the known dispersion matrix we can determine the information matrix for the estimation of treatment effects in model (1). From the paper Markiewicz and Szczepańska (2007) this matrix has the following form

$$
\mathbf{M}_{t, d}=\left(\mathbf{C}_{1, t}^{\prime} \boldsymbol{\Sigma}^{-1} \mathbf{C}_{1, t}\right) \otimes\left(\mathbf{A}_{1, d}^{\prime} \mathbf{Q}_{A_{2}} \mathbf{A}_{1, d}\right),
$$

where $\mathbf{Q}_{A}=\mathbf{I}-\mathbf{A}\left(\mathbf{A}^{\prime} \mathbf{A}\right)^{-1} \mathbf{A}^{\prime}$ is the orthocomplement of the column space of matrix A. Observe that the matrix $\mathbf{C}_{1, t}^{\prime} \boldsymbol{\Sigma}^{-1} \mathbf{C}_{1, t}$ depends only on an allocation of time points in the experiment and is the information matrix for the estimation of $\boldsymbol{\beta}_{1}$ in the following univariate model

$$
\mathbf{y}=\mathbf{C}_{1, t}^{\prime} \boldsymbol{\beta}_{1}+\boldsymbol{\epsilon}, \quad \boldsymbol{\epsilon} \sim N_{q}(\mathbf{0}, \boldsymbol{\Sigma})
$$

where $\mathbf{y} \in I R^{q}, \boldsymbol{\beta}_{1} \in I R^{(p+1)}$ and $\boldsymbol{\epsilon} \in I R^{q}$. The matrix $\mathbf{A}_{1, d}^{\prime} \mathbf{Q}_{A_{2}} \mathbf{A}_{1, d}$ depends on an allocation of treatments in the block design and is the information matrix for the estimation of treatment effects $\left(\boldsymbol{\beta}_{1}\right)$ in the following model in the block design 


$$
\mathbf{y}=\mathbf{A}_{1, d} \boldsymbol{\beta}_{1}+\mathbf{A}_{2} \boldsymbol{\beta}_{2}+\boldsymbol{\epsilon}, \quad \boldsymbol{\epsilon} \sim N_{n}\left(\mathbf{0}, \mathbf{I}_{n}\right),
$$

where $\mathbf{y} \in I R^{n}, \boldsymbol{\beta}_{1} \in \mathbb{R}^{v}, \boldsymbol{\beta}_{2} \in \mathbb{R}^{b}, \boldsymbol{\epsilon} \in \mathbb{I}^{n}$.

Looking for the optimal choice of time points in the experiment in model (1) we assume that the number of time points is equal to the number of regression coefficients. It was showed by Garza (1954) that for the polynomial regression model of degree $p$ with uncorrelated errors the dispersion matrix of the estimated polynomial coefficients can be attained by the spacing the information at only $p+1$ values. The de la Garza phenomenon was used in the theory of optimal designs, for example in papers written by Luoma et al. (2001), Mandal (2002). Moreover, Moerbeek (2005) showed that the efficiency of optimal design with $p+1 \leq q \leq 6$ time points relative to the optimal design with $p+1$ time points and as function of autocorrelation coefficient relative decreases when the number of time point increases in model (4). This property was analyzed using criteria of A-, D- and E-optimality for the estimation of $\boldsymbol{\beta}_{1}$ in model (4). Also Yang (2010) showed that the de la Garza phenomenon exists for many nonlinear models.

Moreover, we assume in the matrix $\mathbf{C}_{1, t}$ that $l_{i}<l_{j}, i, j=1,2, \ldots, q$, $i<j$. Then $\operatorname{det}\left(\mathbf{C}_{1, t}^{\prime} \boldsymbol{\Sigma}^{-1} \mathbf{C}_{1, t}\right)>0$ and from the fact that $\mathbf{C}_{1, t}^{\prime} \boldsymbol{\Sigma}^{-1} \mathbf{C}_{1, t} \in N N D(q)$, where $\operatorname{NND}(q)$ denotes the class of nonnegative definite matrices of order $q$, we have $\mathbf{C}_{1, t}^{\prime} \Sigma^{-1} \mathbf{C}_{1, t} \in P D(q)$, where $P D(q)$ denotes the class of positive definite matrices of order $q$. Moreover, we look for the optimal design with respect to an allocation of treatments in the block in the class of connected designs. Following Caliński and Kageyama (2000) a block design is said to be connected if, for given any two treatments $i$ and $i^{\prime}$, it is possible to construct a chain of treatments $i=i_{0}, i_{1}, \ldots, i_{m}=i^{\prime}$ such that every consecutive two treatments in the chain occur together in a block. The example of the disconnected design and the connected design which fulfils the above definition is given below.

Example (John 1987)

Disconnected Design :

Connected Design :

Design 1: $d_{1}=\left[\begin{array}{cc}A & C \\ B & D \\ C & E \\ D & F \\ E & A \\ F & B\end{array}\right] \quad$ Design $2: d_{2}=\left[\begin{array}{cc}A & B \\ B & C \\ C & D \\ D & E \\ E & F \\ F & A\end{array}\right]$

Chain of Design 1

Chain of Design 2

$A \rightarrow C \rightarrow E$, or $B \rightarrow D \rightarrow F \quad A \rightarrow B \rightarrow C \rightarrow D \rightarrow E \rightarrow F$

In the class of connected designs the rank of information matrix is equal to the number of treatments minus 1 . We assume that $\mathcal{D}$ is the class of connected designs so $\operatorname{rank}\left(\mathbf{A}_{1, d}^{\prime} \mathbf{Q}_{A_{2}} \mathbf{A}_{1, d}\right)=v-1$ for all $d \in \mathcal{D}$. 
Taking into consideration the above properties of matrices $\mathbf{C}_{1, t}^{\prime} \boldsymbol{\Sigma}^{-1} \mathbf{C}_{1, t}$ and $\mathbf{A}_{1, d}^{\prime} \mathbf{Q}_{A_{2}} \mathbf{A}_{1, d}$ the information matrix, $\mathbf{M}_{t, d}$, given in (3), can be written as

$$
\begin{aligned}
\mathbf{M}_{t, d}= & \left(\mathbf{V}_{t} \otimes \mathbf{U}_{d}\right) \in(\operatorname{PD}(q) \otimes \operatorname{NND}(v)) \subset \mathrm{NND}(q v) \\
& \text { and } \operatorname{rank}\left(\mathbf{M}_{t, d}\right)=q v-q,
\end{aligned}
$$

where $\mathbf{V}_{t}=\mathbf{C}_{1, t}^{\prime} \boldsymbol{\Sigma}^{-1} \mathbf{C}_{1, t}, \mathbf{U}_{d}=\mathbf{A}_{1, d}^{\prime} \mathbf{Q}_{A_{2}} \mathbf{A}_{1, d}$.

\section{Optimality criteria}

Let consider optimality criteria given in Pukelsheim (1993) which are based on the information function $\phi$ from the closed cone of nonnegative definite matrices into the real line:

$$
\phi: \mathrm{NND}(u) \rightarrow I R,
$$

where the function $\phi$ is isotonic, concave, nonconstant and positive homogeneous.

Denote by $\boldsymbol{\Phi}$ the class of all information functions and let $\mathcal{G}$ be the class of designs and $\mathbf{W}_{g}$ be the information matrix depended on design $g \in \mathcal{G}$. The definition of optimality of design is as follows:

Definition 1 Design $g^{*}$ is called a $\phi$-optimal design in the class of designs $\mathcal{G}$, if $g^{*}$ maximizes $\phi\left(\mathbf{W}_{g^{*}}\right)$ for any arbitrary function $\phi \in \boldsymbol{\Phi}$.

The aim of the paper is to consider the optimality of designs in two aspects: time and the block design. Let $\chi \in \boldsymbol{\Phi}$ and $\mathbf{F}_{t, d}$ be the information matrix dependent on design $(t, d) \in \mathcal{T} \times \mathcal{D}$, where $\mathcal{T} \times \mathcal{D}$ means the class of pairs of designs: the first from class $\mathcal{T}$ and the second from class $\mathcal{D}$. We determine the optimal design in the new context using the following definition:

Definition 2 Design $\left(t^{*}, d^{*}\right)$ is called a $\chi$-optimal in the class of designs $\mathcal{T} \times \mathcal{D}$ if for all pairs of designs $(t, d) \in \mathcal{T} \times \mathcal{D}$ the following inequality holds

$$
\chi\left(\mathbf{F}_{t, d}\right) \leq \chi\left(\mathbf{F}_{t^{*}, d^{*}}\right)
$$

for any arbitrary function $\chi \in \boldsymbol{\Phi}$.

The classical optimality criteria as the average-variance criterion, the determinant criterion and the smallest-eigenvalue criterion are based on the following information functions 


$$
\tilde{\phi}_{A}(\mathbf{C})=\left(\frac{1}{k} \operatorname{tr} \mathbf{C}^{-1}\right)^{-1}, \quad \tilde{\phi}_{D}(\mathbf{C})=(\operatorname{det} \mathbf{C})^{1 / k}, \quad \tilde{\phi}_{E}(\mathbf{C})=\lambda_{\min }(\mathbf{C})
$$

where $\mathbf{C}$ is the $k \times k$ nonsingular information matrix and $\operatorname{tr}(),. \operatorname{det}(),. \lambda_{\min }($.$) denote$ trace, determinant and the smallest eigenvalue of matrix.

Observe that in the class of connected designs the information matrix $\mathbf{C} \in I R_{k \times k}$ is singular and $\operatorname{rank}(\mathbf{C})=k-1$. Then functions given in (7) are not specified correctly because $\tilde{\phi}_{D}(\mathbf{C}), \tilde{\phi}_{E}(\mathbf{C})$ are equal to zero and $\tilde{\phi}_{A}(\mathbf{C})$ can not be calculated. In this case the first zero eigenvalue of matrix $\mathbf{C}$ should not be taken into account and information functions have to be formulated as follows

$$
\begin{aligned}
\phi_{A}(\mathbf{C})= & \left(\frac{1}{k-1} \sum_{i=1}^{k-1} \lambda_{i}(\mathbf{C})^{-1}\right)^{-1}, \quad \phi_{D}(\mathbf{C})=\left(\prod_{i=1}^{k-1} \lambda_{i}(\mathbf{C})\right)^{1 /(k-1)}, \\
& \phi_{E}(\mathbf{C})=\lambda_{\min }(\mathbf{C}),
\end{aligned}
$$

where $\lambda_{i}(\mathbf{C})$ denotes $i$-th eigenvalue of matrix $\mathbf{C}$ and $\lambda_{\min }(\mathbf{C})$ means the smallest nonzero eigenvalue of matrix $\mathbf{C}$.

In the case, $\mathbf{C}=\mathbf{A} \otimes \mathbf{B}$, where $\mathbf{A} \in P D(p)$ and $\mathbf{B} \in N N D(r), \operatorname{rank}(\mathbf{B})=r-1$, information functions, given in (8), are

$$
\begin{aligned}
\chi_{A}(\mathbf{C})= & \left(\frac{1}{p r-p} \sum_{i=1}^{p r-p} \lambda_{i}(\mathbf{C})^{-1}\right)^{-1}, \chi_{D}(\mathbf{C})=\left(\prod_{i=1}^{p r-p} \lambda_{i}(\mathbf{C})\right)^{1 /(p r-p)}, \\
& \chi_{E}(\mathbf{C})=\lambda_{\min }(\mathbf{C}),
\end{aligned}
$$

where $\lambda_{\min }(\mathbf{C})$ means the smallest nonzero eigenvalue of matrix $\mathbf{C}$.

The examples of $\phi$-optimal designs are A-, D-, E-optimal designs. We say that design $g^{*}$ is A-, D- or E-optimal, if for each design $g \in \mathcal{G}$ the following condition is fulfiled

$$
\tilde{\phi}_{A}\left(\mathbf{W}_{g}\right) \leq \tilde{\phi}_{A}\left(\mathbf{W}_{g^{*}}\right), \quad \tilde{\phi}_{D}\left(\mathbf{W}_{g}\right) \leq \tilde{\phi}_{D}\left(\mathbf{W}_{g^{*}}\right), \quad \tilde{\phi}_{E}\left(\mathbf{W}_{g}\right) \leq \tilde{\phi}_{E}\left(\mathbf{W}_{g^{*}}\right),
$$

respectively. The same criteria are fulfiled when instead of function $\tilde{\phi}$ we use $\phi$ or $\chi$.

\section{Optimal designs}

Based on optimality criteria presented in Sect. 3 we formulate the theorem which characterizes the optimal design with respect to an allocation of time points and an allocation of treatments in the experiment.


of $\mathbf{B}_{1}$ in model (1) if and only if $t^{*} \in \mathcal{T}$ is A-optimal ( $\tilde{\phi}_{A}$-optimal) for the estimation 
of $\boldsymbol{\beta}_{1}$ in model (4) and $d^{*} \in \mathcal{D}$ is A-optimal ( $\phi_{A}$-optimal) for the estimation of $\boldsymbol{\beta}_{1}$ in model (5).

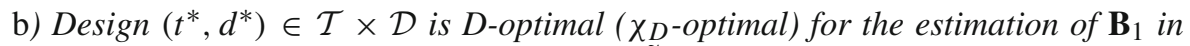
model (1) if and only if $t^{*} \in \mathcal{T}$ is D-optimal $\left(\tilde{\phi}_{D^{-o p t i m a l}}\right.$ ) for the estimation of $\boldsymbol{\beta}_{1}$ in

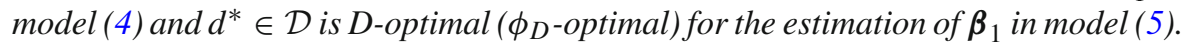

c) Design $\left(t^{*}, d^{*}\right) \in \mathcal{T} \times \mathcal{D}$ is E-optimal ( $\chi_{E}$-optimal) for the estimation of $\mathbf{B}_{1}$ in model (1) if and only if $t^{*} \in \mathcal{T}$ is E-optimal ( $\tilde{\phi}_{E}$-optimal) for the estimation of $\boldsymbol{\beta}_{1}$ in model (4) and $d^{*} \in \mathcal{D}$ is E-optimal ( $\phi_{E}$-optimal) for the estimation of $\boldsymbol{\beta}_{1}$ in model (5).

Proof a) Observe that the information matrix for the estimation of $\mathbf{B}_{1}$ in model (1), given in (6), is singular so to find the optimal design we use information functions given in (9). Let consider matrix $\mathbf{M}_{t, d}=\mathbf{V}_{t} \otimes \mathbf{U}_{d}$ and let $\boldsymbol{\Phi}$ be the class of all information functions and $\chi_{A}, \phi_{A}, \tilde{\phi}_{A} \in \boldsymbol{\Phi}$. From (9) and using properties of Kronecker product we have the following forms

$$
\begin{aligned}
\chi_{A}\left(\mathbf{M}_{t, d}\right) & =\chi_{A}\left(\mathbf{V}_{t} \otimes \mathbf{U}_{d}\right)=\left(\frac{1}{q v-q} \sum_{i=1}^{q v-q} \lambda_{i}\left(\mathbf{V}_{t}^{-1}\right) \cdot \lambda_{i}\left(\mathbf{U}_{d}^{-1}\right)\right)^{-1} \\
& =\left(\frac{1}{q} \sum_{i=1}^{q} \lambda_{i}\left(\mathbf{V}_{t}^{-1}\right)\right)^{-1}\left(\frac{1}{v-1} \sum_{i=1}^{v-1} \lambda_{i}\left(\mathbf{U}_{d}^{-1}\right)\right)^{-1}=\tilde{\phi}_{A}\left(\mathbf{V}_{t}\right) \cdot \phi_{A}\left(\mathbf{U}_{d}\right)
\end{aligned}
$$

Observe that from Definition 2 we get

$$
\max _{(t, d) \in \mathcal{T} \times \mathcal{D}} \chi_{A}\left(\mathbf{M}_{t, d}\right)=\max _{(t, d) \in \mathcal{T} \times \mathcal{D}}\left(\tilde{\phi}_{A}\left(\mathbf{V}_{t}\right) \cdot \phi_{A}\left(\mathbf{U}_{d}\right)\right)=\max _{t \in \mathcal{T}} \tilde{\phi}_{A}\left(\mathbf{V}_{t}\right) \cdot \max _{d \in \mathcal{D}} \phi_{A}\left(\mathbf{U}_{d}\right) .
$$

Design $\left(t^{*}, d^{*}\right)$ which maximizes the above form in the class of design $\mathcal{T} \times \mathcal{D}$ is optimal in model (1) so the same designs $t^{*}$ and $d^{*}$ are also optimal in models (4) and (5).

The proofs of (b) and (c) run similar as in (a).

Theorem 1 shows that planning the experiment with the optimal choice of time points and the optimal allocation of treatments in the block design which is modeled by the growth curve model it is enough to choose optimal designs (in terms of the same optimality criteria) in adequate univariate models. The optimal designs in univariate model in the block design, model (5), were proposed among others by Cheng (1978, 1980), Hedayat and Li (1979), Gaffke (1982), Majumar and Notz (1983), John (1987), Azais et al. (1993), Srivastav and Shankar (2003) and the optimal choice of time points in model (4) was determined by Abt et al. (1998), Imhof (1998), Gaffke and Heiligers (1998), Luoma et al. (2001), Chang and Lay (2002), Mandal (2002), Moerbeek (2005) and others. Some examples of optimal designs in the growth curve model are given below. 
Design $\left\{t_{A}^{*}, d_{A}^{*}\right\}$, where $t_{A}^{*}=\{0,0.2516,0.7479,1\}$ (Luoma et al. 2001),

$$
d_{A}^{*}=\left[\begin{array}{ccc}
B & C & D \\
A & D & C \\
D & A & B \\
C & B & A
\end{array}\right] \text { (Azais et al. 1993) }
$$

is A-optimal in model (1) with $p=3$ and $\boldsymbol{\Sigma}=\mathbf{I}_{4}$. Time points are chosen from time interval $[0,1]$. Rows of $d_{A}^{*}$ mean blocks and treatments are denoted by $A, B, C, D$. Design $\left\{t_{D}^{*}, d_{D}^{*}\right\}$, where $t_{D}^{*}=\{-1,-0.602,0.602,1\}$ (Luoma et al. 2001)

$$
d_{D}^{*}=\left[\begin{array}{ccc}
A & B & D \\
B & C & E \\
C & D & F \\
D & E & G \\
E & F & A \\
F & G & B \\
G & A & C
\end{array}\right] \text { (Hedayat and Li 1979) }
$$

is D-optimal in model (1) with $p=3$ and $\boldsymbol{\Sigma}=\mathbf{I}_{4}$. Time points are chosen from time interval $[-1,1]$. Rows of $d_{D}^{*}$ mean blocks and treatments are denoted by $A, B, C, D, E, F, G$.

The design $\left\{t_{E}^{*}, d_{E}^{*}\right\}$, where $t_{E}^{*}=\{0,1.069,2\}$ (Moerbeek 2005),

$$
d_{E}^{*}=\left[\begin{array}{ccc}
A & B & G \\
A & C & H \\
A & D & B \\
A & E & C \\
A & F & D \\
B & C & F \\
B & E & H \\
C & D & G \\
D & E & H \\
E & F & G \\
F & G & H
\end{array}\right] \text { (Srivastav and Shankar 2003) }
$$

is E-optimal in model (1) with $p=2$ and $\boldsymbol{\Sigma}=\mathbf{I}_{3}$. Time points are chosen from time interval $[0,2]$. Rows of $d_{E}^{*}$ mean blocks and treatments are denoted by $A, B, C, D, E, F, H$.

\section{Some relations for information functions for different designs}

In this section we show some relations between information functions for different optimality criteria. Let $\geq_{L}$ denote Loewner ordering. Remember that $\mathbf{A} \geq_{L} \mathbf{B}$ if and only if $\mathbf{A}-\mathbf{B}$ is the nonnegative definite matrix. Let $\mathbf{W}_{g_{1}}$ and $\mathbf{W}_{g_{2}}$ be the information 
matrices under designs $g_{1}$ and $g_{2}$, respectively. It is said that design $g_{1}$ dominates design $g_{2}, g_{1} \succ g_{2}$, if $\mathbf{W}_{g_{1}} \geq_{L} \mathbf{W}_{g_{2}}$. Unfortunately, using Loewner ordering, the optimal design in the given class can not be found. Pukelsheim (1993) showed that there no exists optimal design which dominates in Loewner ordering sense all designs from given class. Theorem 2 shows some relations between information functions for different designs in models (1), (4), and (5) under assumptions $t_{1} \succ t_{2}, d_{1} \succ d_{2}$.

Theorem 2 Let $\mathbf{V}_{t_{1}} \in I R_{q \times q}$ and $\mathbf{V}_{t_{2}} \in I R_{q \times q}$ be the information matrices for the estimation of $\boldsymbol{\beta}_{1}$ in model (4) for two different designs $t_{1}, t_{2} \in \mathcal{T}$ and $\mathbf{U}_{d_{1}} \in I R_{v \times v}$ and $\mathbf{U}_{d_{2}} \in I R_{v \times v}$ be information matrices for the estimation of $\boldsymbol{\beta}_{1}$ in model (5) for two different designs $d_{1}, d_{2} \in \mathcal{D}$, where $\mathcal{D}$ is the class of connected designs. Moreover, let $\mathbf{V}_{t_{1}} \otimes \mathbf{U}_{d_{1}} \in I R_{v q \times v q}$ be the information matrix for the estimation of $\mathbf{B}_{1}$ in model (1) for the design $\left(t_{1}, d_{1}\right)$. If $\mathbf{V}_{t_{1}} \geq_{L} \mathbf{V}_{t_{2}}$ and $\mathbf{U}_{d_{1}} \geq_{L} \mathbf{U}_{d_{2}}$ then

a)

$$
\begin{gathered}
\chi_{D}\left(\mathbf{V}_{t_{1}} \otimes \mathbf{U}_{d_{1}}\right) \geq\left(\varphi_{E}\left(\mathbf{V}_{t_{2}}\right)\right)^{1 /(v-1)} \cdot\left(\varphi_{D}\left(\mathbf{U}_{d_{2}}\right)\right)^{1 / q} \\
\text { or } \quad \chi_{D}\left(\mathbf{V}_{t_{1}} \otimes \mathbf{U}_{d_{1}}\right) \geq\left(\varphi_{D}\left(\mathbf{V}_{t_{2}}\right)\right)^{1 /(v-1)} \cdot\left(\varphi_{E}\left(\mathbf{U}_{d_{2}}\right)\right)^{1 / q}
\end{gathered}
$$

b)

$$
\chi_{A}\left(\mathbf{V}_{t_{1}} \otimes \mathbf{U}_{d_{1}}\right) \geq \varphi_{E}\left(\mathbf{V}_{t_{2}}\right) \cdot \varphi_{A}\left(\mathbf{U}_{d_{2}}\right) \quad \text { or } \quad \chi_{A}\left(\mathbf{V}_{t_{1}} \otimes \mathbf{U}_{d_{1}}\right) \geq \varphi_{A}\left(\mathbf{V}_{t_{2}}\right) \cdot \varphi_{E}\left(\mathbf{U}_{d_{2}}\right)
$$

Proof Observe that from assumptions given in the theorem we have

$\mathbf{V}_{t_{1}} \geq_{L} \mathbf{V}_{t_{2}}, \mathbf{U}_{d_{1}} \geq_{L} \mathbf{U}_{d_{2}}$. These relations imply that nonnegative definite matrices, $\mathbf{P}$ and $\mathbf{R}$, exist and $\overline{\mathbf{V}}_{t_{1}}=\mathbf{V}_{t_{2}}+\mathbf{P}$ and $\mathbf{U}_{d_{1}}=\mathbf{U}_{d_{2}}+\mathbf{R}$.

Let consider Kronecker product of matrices $\mathbf{V}_{t_{1}}$ and $\mathbf{U}_{d_{1}}$,

$$
\begin{aligned}
\mathbf{V}_{t_{1}} \otimes \mathbf{U}_{d_{1}} & =\left(\mathbf{V}_{t_{2}}+\mathbf{P}\right) \otimes \mathbf{U}_{d_{1}}=\mathbf{V}_{t_{2}} \otimes \mathbf{U}_{d_{1}}+\mathbf{P} \otimes \mathbf{U}_{d_{1}}=\mathbf{V}_{t_{2}} \otimes\left(\mathbf{U}_{d_{2}}+\mathbf{R}\right)+\mathbf{P} \otimes \mathbf{U}_{d_{1}} \\
& =\mathbf{V}_{t_{2}} \otimes \mathbf{U}_{d_{2}}+\mathbf{V}_{t_{2}} \otimes \mathbf{R}+\mathbf{P} \otimes \mathbf{U}_{d_{1}}
\end{aligned}
$$

The matrix $\mathbf{V}_{t_{2}} \otimes \mathbf{R}+\mathbf{P} \otimes \mathbf{U}_{d_{1}}$ is nonnegative definite so we get

$$
\mathbf{V}_{t_{1}} \otimes \mathbf{U}_{d_{1}} \geq_{L} \mathbf{V}_{t_{2}} \otimes \mathbf{U}_{d_{2}} \Leftrightarrow \mathbf{V}_{t_{1}} \otimes \mathbf{U}_{d_{1}}-\mathbf{V}_{t_{2}} \otimes \mathbf{U}_{d_{2}} \in \mathrm{NND}(q v)
$$

Based on Theorem 3.18 given by Schott (1997) and from (10) we obtain

$$
\lambda_{i}\left(\mathbf{V}_{t_{1}} \otimes \mathbf{U}_{d_{1}}\right) \geq \lambda_{i}\left(\mathbf{V}_{t_{2}} \otimes \mathbf{U}_{d_{2}}\right), \quad i=1,2, \ldots, q v
$$

Let take only positive eigenvalues of matrices $\mathbf{V}_{t_{1}} \otimes \mathbf{U}_{d_{1}}, \mathbf{V}_{t_{2}} \otimes \mathbf{U}_{d_{2}}$ then

$$
\lambda_{i}\left(\mathbf{V}_{t_{1}} \otimes \mathbf{U}_{d_{1}}\right) \geq \lambda_{i}\left(\mathbf{V}_{t_{2}} \otimes \mathbf{U}_{d_{2}}\right), \quad i=1,2, \ldots, q v-q .
$$

(a) From (11) we have

$$
\prod_{i=1}^{q v-q} \lambda_{i}\left(\mathbf{V}_{t_{1}} \otimes \mathbf{U}_{d_{1}}\right) \geq \prod_{i=1}^{q v-q} \lambda_{i}\left(\mathbf{V}_{t_{2}} \otimes \mathbf{U}_{d_{2}}\right)
$$




$$
\prod_{i=1}^{q} \prod_{j=1}^{v-1} \lambda_{i}\left(\mathbf{V}_{t_{1}}\right) \lambda_{j}\left(\mathbf{U}_{d_{1}}\right) \geq \prod_{i=1}^{q} \prod_{j=1}^{v-1} \lambda_{i}\left(\mathbf{V}_{t_{2}}\right) \lambda_{j}\left(\mathbf{U}_{d_{2}}\right) \geq\left(\lambda_{\min }\left(\mathbf{V}_{t_{2}}\right)\right)^{q} \prod_{j=1}^{v-1} \lambda_{j}\left(\mathbf{U}_{d_{2}}\right)
$$

where $\lambda_{\min }\left(\mathbf{V}_{t_{2}}\right)$ is the smallest eigenvalue of matrix $\mathbf{V}_{t_{2}}$. Taking the above expression to the power $\frac{1}{q v-q}$ we have

$$
\begin{gathered}
\left(\prod_{i=1}^{q} \prod_{j=1}^{v-1} \lambda_{i}\left(\mathbf{V}_{t_{1}}\right) \lambda_{j}\left(\mathbf{U}_{d_{1}}\right)\right)^{1 /(q v-q)} \geq\left(\lambda_{\min }\left(\mathbf{V}_{t_{2}}\right)\right)^{1 /(v-1)}\left(\left(\prod_{j=1}^{v-1} \lambda_{j}\left(\mathbf{U}_{d_{2}}\right)\right)^{1 /(v-1)}\right)^{1 / q}, \\
\chi_{D}\left(\mathbf{V}_{t_{1}} \otimes \mathbf{U}_{d_{1}}\right) \geq\left(\phi_{E}\left(\mathbf{V}_{t_{2}}\right)\right)^{1 /(v-1)}\left(\phi_{D}\left(\mathbf{U}_{d_{2}}\right)\right)^{1 / q} .
\end{gathered}
$$

In the same manner we can see that

$$
\chi_{D}\left(\mathbf{V}_{t_{1}} \otimes \mathbf{U}_{d_{1}}\right) \geq\left(\varphi_{D}\left(\mathbf{V}_{t_{2}}\right)\right)^{1 /(v-1)} \cdot\left(\varphi_{E}\left(\mathbf{U}_{d_{2}}\right)\right)^{1 / q}
$$

(b) Similar arguments to (a) apply to case (b).

Let take nonzero eigenvalues of matrices $\mathbf{V}_{t_{1}} \otimes \mathbf{U}_{d_{1}}$ and $\mathbf{V}_{t_{2}} \otimes \mathbf{U}_{d_{2}}$. Then

$$
\left(\lambda_{i}\left(\mathbf{V}_{t_{1}}\right) \lambda_{j}\left(\mathbf{U}_{d_{1}}\right)\right)_{k} \geq\left(\lambda_{i}\left(\mathbf{V}_{t_{2}}\right) \lambda_{j}\left(\mathbf{U}_{d_{2}}\right)\right)_{k}, \quad k=1,2, \ldots, q v-q
$$

where $\left(\lambda_{i}(\mathbf{A}) \lambda_{j}(\mathbf{B})\right)_{k}$ denotes $k$-th positive eigenvalue of matrix $\mathbf{A} \otimes \mathbf{B}$.

$$
\begin{gathered}
\frac{1}{\left(\lambda_{i}\left(\mathbf{V}_{t_{1}}\right) \lambda_{j}\left(\mathbf{U}_{d_{1}}\right)\right)_{k}} \leq \frac{1}{\left(\lambda_{i}\left(\mathbf{V}_{t_{2}}\right) \lambda_{j}\left(\mathbf{U}_{d_{2}}\right)\right)_{k}}, \quad k=1,2, \ldots, q v-q . \\
\sum_{k=1}^{q v-q} \frac{1}{\left(\lambda_{i}\left(\mathbf{V}_{t_{1}}\right) \lambda_{j}\left(\mathbf{U}_{d_{1}}\right)\right)_{k}} \leq \sum_{k=1}^{q v-q} \frac{1}{\left(\lambda_{i}\left(\mathbf{V}_{t_{2}}\right) \lambda_{j}\left(\mathbf{U}_{d_{2}}\right)\right)_{k}} \leq \frac{1}{\lambda_{\min }\left(\mathbf{V}_{t_{2}}\right)} \sum_{j=1}^{v-1} \frac{q}{\lambda_{j}\left(\mathbf{U}_{d_{2}}\right)}
\end{gathered}
$$

Multiplying the inequality by $\frac{1}{q v-q}$ and inverting the above form we get

$$
\begin{gathered}
\left(\frac{1}{q v-q} \sum_{k=1}^{q v-q} \frac{1}{\left(\lambda_{i}\left(\mathbf{V}_{t_{1}}\right) \lambda_{j}\left(\mathbf{U}_{d_{1}}\right)\right)_{k}}\right)^{-1} \geq \lambda_{\min }\left(\mathbf{V}_{t_{2}}\right)\left(\frac{1}{v-1} \sum_{j=1}^{v-1} \frac{1}{\lambda_{j}\left(\mathbf{U}_{d_{2}}\right)}\right)^{-1} \\
\chi_{A}\left(\mathbf{V}_{t_{1}} \otimes \mathbf{U}_{d_{1}}\right) \geq \varphi_{E}\left(\mathbf{V}_{t_{2}}\right) \cdot \varphi_{A}\left(\mathbf{U}_{d_{2}}\right)
\end{gathered}
$$

Using the same method we can show

$$
\chi_{A}\left(\mathbf{V}_{t_{1}} \otimes \mathbf{U}_{d_{1}}\right) \geq \varphi_{A}\left(\mathbf{V}_{t_{2}}\right) \cdot \varphi_{E}\left(\mathbf{U}_{d_{2}}\right)
$$

The example of designs which fulfil Theorems 2 is given below. 
Example Let $t_{1}=\{0,1.069,2\}$ and $t_{2}=\{0,0.9,2\}$ be designs in model (4) with $p=2, \boldsymbol{\Sigma}=\mathbf{I}_{3}$, where the time domain is $[0,2]$. Information matrices for the estimation of treatment effects and eigenvalues of these matrices are following

$\mathbf{V}_{t_{1}}=\left[\begin{array}{ccc}1 & 1 & 1 \\ 1 & 3.44866 & 7.70904 \\ 1 & 7.70904 & 21\end{array}\right], \quad$ Eigenvalues $\left[\mathbf{V}_{t_{1}}\right]=\{23.9774,1.3487,0.122517\}$

$$
\mathbf{V}_{t_{2}}=\left[\begin{array}{ccc}
1 & 1 & 1 \\
1 & 2.4661 & 6.04 \\
1 & 6.04 & 21
\end{array}\right], \quad \text { Eigenvalues }\left[\mathbf{V}_{t_{2}}\right]=\{22.8654,1.48529,0.115436\}
$$

respectively.

Let

$$
d_{1}=\left[\begin{array}{lll}
A & C & B \\
A & B & C \\
A & C & B
\end{array}\right], \quad d_{2}=\left[\begin{array}{lll}
A & B & A \\
C & A & C \\
A & B & C
\end{array}\right]
$$

be block designs in the class of designs with $v=b=k=3$. Information matrices for the estimation of treatment effects in model (5) and eigenvalues of these matrices are

$$
\begin{gathered}
\mathbf{U}_{d_{1}}=\left[\begin{array}{rrr}
2 & -1 & -1 \\
-1 & 2 & -1 \\
-1 & -1 & 2
\end{array}\right], \quad \text { Eigenvalues }\left[\mathbf{U}_{d_{1}}\right]=\{3,3,0\}, \\
\mathbf{U}_{d_{2}}=\left[\begin{array}{rrr}
2 & -1 & -1 \\
-1 & \frac{4}{3} & -\frac{1}{3} \\
-1 & -\frac{1}{3} & \frac{4}{3}
\end{array}\right], \quad \text { Eigenvalues }\left[\mathbf{U}_{d_{2}}\right]=\{3,1.66667,0\},
\end{gathered}
$$

respectively.

Matrices $\mathbf{V}_{t_{1}}, \mathbf{V}_{t_{2}}, \mathbf{U}_{d_{1}}$ and $\mathbf{U}_{d_{2}}$ fulfil following assumptions $\mathbf{V}_{t_{1}} \geq_{L} \mathbf{V}_{t_{2}}$ and $\mathbf{U}_{d_{1}} \geq_{L} \mathbf{U}_{d_{2}}$. Let $\mathbf{V}_{t_{1}} \otimes \mathbf{U}_{d_{1}}$ be the information matrix for the estimation of $\mathbf{B}_{1}$ in model (1) for design $\left(t_{1}, d_{1}\right)$. Moreover,

$$
\begin{aligned}
& \chi_{D}\left(\mathbf{V}_{t_{1}} \otimes \mathbf{U}_{d_{1}}\right)=4.74708, \quad\left(\varphi_{E}\left(\mathbf{V}_{t_{2}}\right)\right)^{1 / 2}=0.339759, \quad\left(\varphi_{D}\left(\mathbf{U}_{d_{2}}\right)\right)^{1 / 3}=1.30766 \\
& \left(\varphi_{D}\left(\mathbf{V}_{t_{2}}\right)\right)^{1 / 2}=1.25571, \quad\left(\varphi_{E}\left(\mathbf{U}_{d_{2}}\right)\right)^{1 / 3}=1.18563
\end{aligned}
$$

and

$$
\begin{gathered}
\chi_{A}\left(\mathbf{V}_{t_{1}} \otimes \mathbf{U}_{d_{1}}\right)=1.00612, \quad \varphi_{E}\left(\mathbf{V}_{t_{2}}\right)=0.115436, \quad \varphi_{A}\left(\mathbf{U}_{d_{2}}\right)=2.14286 \\
\varphi_{A}\left(\mathbf{V}_{t_{2}}\right)=0.319836, \quad \varphi_{E}\left(\mathbf{U}_{d_{2}}\right)=1.66667
\end{gathered}
$$


Now, it is easy to see that given designs fulfil relations from Theorems 2.

Observe that by formulating a proof similar to the proof of the second formula of Theorem 2, part b, we can show relations given in the following corollary.

Corollary 1 If $t_{1}=t_{2}=t$ and $\mathbf{U}_{d_{1}} \geq_{L} \mathbf{U}_{d_{2}}$ then $\phi_{A}\left(\mathbf{U}_{d_{1}}\right) \geq \phi_{E}\left(\mathbf{U}_{d_{2}}\right)$. If $d_{1}=d_{2}=d$ and $\mathbf{V}_{t_{1}} \geq_{L} \mathbf{V}_{t_{2}}$ then $\tilde{\phi}_{A}\left(\mathbf{V}_{t_{1}}\right) \geq \tilde{\phi}_{E}\left(\mathbf{V}_{t_{2}}\right)$.

\section{Conclusion}

It was shown in Sect. 4 that designs which are optimal in the growth curve model are also optimal in the appropriate univariate model with respect to the same optimality criteria. Properties of determinant, trace and eigenvalues of Kronecker product of two matrices facilitate the determination of optimal design in model (1). It is also interesting to find the optimal design in the growth curve model using mixed criteria of optimality. This problem is more complex and to solve it the analyse of properties of information function is needed. The function which is the product of two arbitrary information functions is not the information function. To find the optimal design in the growth curve model with respect to mixed optimality criteria probably the invention of new functional should be considered. It opens the possibility of future research.

Acknowledgement The author would like to thank the referees for valuable comments and suggestions.

Open Access This article is distributed under the terms of the Creative Commons Attribution License which permits any use, distribution, and reproduction in any medium, provided the original author(s) and the source are credited.

\section{References}

Abt M, Gaffke N, Liski EP, Sinha BK (1998) Optimal designs in growth curve models-II, correlated model for quadratic growth: optimal designs for parameter estimation and growth prediction. J Stat Plan Inference 67:287-296

Azais J-M, Bailey RA, Mond H (1993) The consultant's forum. A catalogue of efficient neighbour-design with boder plots. Biometrics 49:1252-1261

Caliński T, Kageyama S (2000) Block designs: a randomization approach, vol I. Springer-Verlag, New York Chang F-C, Lay C-F (2002) Optimal designs for growth curve models. J Stat Plan Inference 104:427-438 Cheng C-S (1978) Optimality of certain asymmetrical experimental designs. Ann Stat 6(6):1239-1261

Cheng C-S (1980) On the E-optimality of some block design. J R Stat Soc B 42(2):199-204

de la Garza A (1954) Spacing of information in polynomial regression. Ann Math Stat 25:123-130

Filipiak K, Markiewicz A, Szczepańska A (2009) Optimal designs under a multivariate linear model with additional nuisance parameters. Stat Papers 50:761-778

Filipiak K, Szczepańska A (2005) A-, D- and E-optimal designs for quadratic and cubic growth curve models with correlated errors. Biom Lett 42/1:43-56

Filipiak K, Szczepańska A (2007) A-optimal designs under a quadratic growth curve model in the transformed time interval. Biom Lett 44/2:85-96

Gaffke N (1982) D-optimal block designs with at most six varieties. J Stat Plan Inference 6:183-200

Gaffke N, Heiligers B (1998) Minimum support invariant design for multiple cubic regression. J Stat Plan Inference 72:229-245 
Hedayat A, Li S-Y (1979) The trade-off method in the construction of BIB designs with variable support size. Ann Stat 7(6):1277-1287

Imhof LA (1998) A-optimum extract design for quadratic regresion. J Math Anal Appl 228:157-165

John JA (1987) Cyclic designs. Chapman and Hall, New York

Luoma A, Mandal NK, Sinha BK (2001) A-optimal cubic and quartic regression designs in asymmetric factor spaces. Stat Appl 3:147-153

Majumar D, Notz WI (1983) Optimal incomplete block design for comparing treatments with a control. Ann Stat 11(1):258-266

Mandal NK (2002) Optimal regression design in symmetric domains. Design workshop lecture notes. ISI, Kolkata, pp 23-32

Markiewicz A, Szczepańska A (2007) Optimal designs for multivariate linear models. Stat Probab Lett $77: 426-430$

Moerbeek M (2005) Robustness properties of A-, D-, and E-optimal designs for polynomial growth models with autocorrelated errors. Comput Stat Data Anal 48:765-778

Potthoff RF, Roy SN (1964) A generalized multivariate analysis of variance model useful especially for growth curve problems. Biometrika 51:313-326

Pukelsheim F (1993) Optimal designs of experiments. Wiley, New York

Schott JR (1997) Analysis for statistics. Wiley, Toronto

Srivastav SK, Shankar A (2003) On the E-optimality of certain class of block design. Stat Probab Lett 65:187-193

Yang M (2010) On the de la Garza phenomenon. Ann Stat 38:2499-2524 